\section{Rheumatoide Arthritis: wie sind Patienten in ihren Herkunftsländern abgesichert?}

Putrik P et al. Patients with rheumatoid arthritis facing sick leave or work disability meet varying regulations: a study among rheumatologists and patients from 44 European countries. Ann Rheum Dis 2019; 78: 1472-1479

In Europa bestehen große Unterschiede hinsichtlich Krankschreibung und Arbeitsunfähigkeit bei rheumatoider Arthritis, die nicht durch die Heterogenität der Sozialsysteme erklärbar sind. Daher wurden individuelle und länderspezifische sozioökonomische Determinanten des Krankheitsverlaufs sowie die Wahrnehmung der Leistung der Systeme durch Rheumatologen und $\mathrm{Pa}$ tienten in verschiedenen europäischen Ländern bestimmt.

In der von der Maastrichter Universität initiierten Untersuchung wurden 3 Querschnittserhebungen in 50 europäischen Ländern durchgeführt. Eine zur Arbeitsintegration und zum System der sozialen Absicherung bei Krankheit und Langzeit- Arbeitsunfähigkeit aufgrund der rheumatoiden Arthritis (RA). Dazu wurde in 50 europäischen Ländern jeweils ein Rheumatologe pro Land gebeten einen Fragebogen zu den formalen Bedingungen auszufüllen. In 2 weiteren Untersuchungen wurden etwa 15 Rheumatologen und 15 Patienten pro Land gebeten die Absicherung bei Krankheit sowie die Arbeitsintegration in ihrem Land einzuschätzen. Die Unterschiede hinsichtlich Regularien und Wahrnehmungen wurden kategorienübergreifend miteinander verglichen. Die Kategorien waren nach dem Bruttoinlandsprodukt (BIP), der Art des Sozialhilfesystems, der Mitgliedschaft in der Europäischen Union (EU) und dem Verhältnis von RA zu Arbeitsunfähigkeit definiert.

Insgesamt 44 Länder, davon 28 EU- und 16 nicht EU-Mitgliedsstaaten (88\%) lieferten Daten zur Querschnittserhebung hinsichtlich der formalen Voraussetzungen der sozialen Absicherung im Krankheitsfall und der Arbeitsunfähigkeit. Von diesen Ländern sammelten 33 (75\%) Daten von Rheumatologen $(n=539)$ und 34 (77\%) Daten von Patienten ( $n=719)$. Bei den fehlenden Ländern handelte es sich, bis auf Luxemburg, um nicht EU-Staaten.

Verglichen wurden die Regularien der folgenden Sozialsysteme zur Unterstützung von Arbeitsintegration sowie Absicherung von Arbeitsunfähigkeit: angelsächsischer Sozialstaat, Sozialsystem nach dem skandinavischen Modell, das gesetzliche Sozialversicherungswesen nach Bismarck, das mediterrane Sozialsystem sowie das postkommunistische Sozialsystem. Während große Unterschiede zwischen allen Vorschriften in den einzelnen Ländern beobachtet wurden, wurde kein Zusammenhang zwischen den meisten Vorschriften oder Einkommen und dem BIP, der Art der sozialen Absicherung im Krankheitsfall oder den Arbeitsunfähigkeitsquoten gefunden. Rheumatologen in Ländern mit hohem Bruttoinlandsprodukt und in den EU-Mitgliedstaaten nahmen ihre Rolle im Entscheidungsprozess hinsichtlich der Arbeitsunfähigkeit eher gering war. Die höchste Zufriedenheit der Patienten hinsichtlich Bedeutung und Unterstützung des Erhalts der Arbeitsfähigkeit, Beantragung der Arbeitsunfähigkeit sowie Leben mit einer Arbeitsunfähigkeitsrente wurden im skandinavischen und bismarckschen Sozialsystem erzielt. Skandinavisches und bismarcksches Sozialsystem erzielten auch bei den Rheumatologen hinsichtlich der Rolle der Rheumatologen und der Leistung des Systems die besten Ergebnisse.

FAZIT

Die Sozialsysteme in Europa unterscheiden sich erheblich hinsichtlich der Arbeitsunfähigkeit von Patienten mit rheumatoider Arthritis. Nach Meinung der Autoren ist es ein Umstand, der eine Harmonisierung der Strategien gegenüber diesen Patienten erfordert, da sie auf Grund ihrer Erkrankung unter Restriktionen, hinsichtlich der Teilnahme am Arbeitsleben, leiden.

Richard Kessing, Zeiskam 